\title{
Looking for Intermediate-Mass Black Holes
}

\author{
Paul H. Frampton \\ Department of Physics and Astronomy, Univeristy of North Carolina, Chapel Hill, NC 27599-3255.
}

\begin{abstract}
A discussion of the entropy of the universe leads to the suggestion of very many intermediate-mass black holes between thirty and three hundred thousand solar masses in the halo. It is consistent with observations on wide binaries as well as microlensing and considerations of disk stability that such IMBHs constitute all cold dark matter.
\end{abstract}

Keywords: Dark matter, Black holes, Binaries

PACS: $98.80 . E s$

\section{INTRODUCTION}

The present note proposes a solution for the cosmological dark matter problem. The problem is over 75 years old[1] and has been the subject of innumerable papers, conferences and books.

There is about six times more dark matter than baryonic matter in the universe. A wellknown book[2] on the connection between particle phenomenology and theoretical cosmology discusses possible candidates - axions, WIMPs, MACHOs - for the constituents of the dark matter. In the present article I shall provide a complete solution to the dark matter problem which is in the MACHO category and surprisingly simple: the dark mattter is all black holes!

Let me first consider low mass dark matter candidates suggested by particle phenomenology. There are two especially popular candidates.

The lighter is the invisible axion predicted by [3, 4, 5, 6]. Its mass ${ }^{1}$ must be in an allowed window $10^{-15} \mathrm{GeV}<M_{\text {axion }}<10^{-12} \mathrm{GeV}$. Although there are compelling arguments[7] that the invisible axion requires stronger fine tuning than the strong CP problem it purports to solve, it has staying power and remains the subject of experimental searches.

The other very popular low-mass dark matter candidate, at a proposed mass typcally a trillion times that of the invisible axion, is the WIMP (Weakly-Interacting Massive Particle) exemplified by the neutralino of supersymmetry. The fact that WIMPs can naturally annihilate to an acceptable present abundance of dark matter is sometimes cited as support for WIMPs as dark matter.

But if my proposal for identification of the constituents of dark matter as black holes with masses above $30 M_{\odot}$ is correct, particle-phenomenology-inspired dark matter candidates are, unfortunately for their creators, irrelevant.

1 Throughout we adopt an order-of-magnitude notation where $10^{x}$ denotes any integer between $10^{x-1}$ and $10^{x+1}$. 
Higher mass dark matter constituents are generically called MACHOs (Massive Astrophysical Compact Halo Objects). My proposed constituents fall into this class with masses in the range $30 M_{\odot}<M_{M A C H O}<500 M_{\odot}$ where $M_{\odot}$ is the solar mass; equivalently $10^{26} \mathrm{~kg}<M_{\text {МАCHO }}<10^{27} \mathrm{~kg}$ or $10^{55} \mathrm{GeV}<M_{\text {MACHO }}<10^{56} \mathrm{GeV}$. Even the most die-hard opponent to the Large Hadron Collider (LHC) could not take seriously that the LHC could create a black hole with fifty orders of magnitude times the available LHC energy of $10^{4} \mathrm{GeV}$ so the present article thus might convince that the LHC is safe.

The MACHOs are truly compact, all being smaller in physical size than the Earth. It is the small size and large mass which have enabled these dark matter constituents to escape detection for 75 years. We discuss two methods for their detection, their formation then what, for me, is the best motivation - the entropy of the universe.

\section{MACHO SEARCHES BY MICROLENSING}

In the late twentieth century heroic searches were made for microlensing events and many spectacular examples $[8,9]$ of MACHOs were identified with masses up to $30 M_{\odot}$. The length of time of a microlensing event depends on the velocity and mass of the lensing MACHO. Since the velocities do nor vary widely it is the MACHO mass which is most crucial in determining longevity.

The longevity is directly proportional to the square root of the lens mass. MACHOS were discovered with microlensing longevities up to about 400 days. Insufficient MACHOs to account for more than a few percent of the dark matter were discovered. To detect MACHOs up to mass $500 M_{\odot}$, the current upper limit for halo black holes, necessitates study of microlensing events with duration up to 4.5 years.

\section{WIDE BINARIES}

Nature has provided delicate astrophysical clocks whose accuracy is sensitive to the possible proximity of MACHOs. These clocks are binaries with separations up to $1 \mathrm{pc}$ and therefore can be bigger than the Solar System in which the distance from the Sun to the outermost planet is only $10^{-4} \mathrm{pc}$.

Because these wide binaries are so weakly bound they are the most sensitive to disruption by nearby MACHOs. After formation they retain their original orbital parameters as a reliable clock except when affected by gravitational encounters with MACHOs.

The detailed study of wide binaries is a young and promising technique for MACHO detection. In the first pioneering presentation[10] the range of masses allowed for MA$\mathrm{CHOs}$ which provide all dark matter was $30 M_{\odot}<M_{M A C H O}<43 M_{\odot}$. It seemed unlikely, though logically possible, that all dark matter MACHOs could have masses within such a narrow range.

A revisit and reanalysis[11] of wide binaries has, however, recently discovered that one of the key examples used in [10] was spurious. This leads to quite different and much more promising restrictions on MACHOs, with the allowed mass range extending up to $500 M_{\odot}$. It is now far more likely that all dark matter is in the form of black hole MACHOs. 


\section{FORMATION}

Because intergalactic dust shows evidence of metallicity which involves nuclei heavier than lithium, the heaviest element formed during big-bang nucleosynthsis, it has been hypothesized [12] that there must have existed Pop III stars in an era before galaxy formation. These subsequently exploded producing the dust nuclei heavier than lithium, and leaving massive black holes which are MACHO candidates.

Numerical simulations of dark matter halos[13] have given insight into the likely general features of the halo profile. The spatial resolution of such simulations is, however, far too coarse to study the formation of MACHOs smaller than the Earth whose radius is $10^{-10} p c$. Nevertheless, dark matter is known to clump at large scales and may well do so at such far smaller scales.

\section{IRRELEVANCE OF DARK ENERGY}

I have assumed that dark energy possesses no entropy. Let me argue that this is a very weak assumption.

Recently the Planck satellite was launched and is on its way to Lagrangian point 2 where it will attempt to measure with unprecedented accuracy the dark energy equation of state $w=p / \rho$ where $p, \rho$ are respectively pressure, density.

The key quantity is $\phi=(1+w)$. If $\phi$ vanishes identically the assumption of zero entropy for dark energy is justified because it is fully described by one parameter, the cosmological constant. If non-zero $\phi$ were observationally established, it would imply a dynamical dark energy with concomitant entropy. However, without black holes, the dark energy dimensionless entropy would be much less than one googol and so the identification of the dark matter as black holes and the rest of the present discussion would be unchanged.

\section{DISCUSSION}

The identification of the dark matter constituent has been an intellectual pursuit for over 75 years. Particle theorists have naturally suggested light mass solutions which include the invisible axion and WIMPs. According to theoretical cosmology, however, the most likely solution is that all dark matter is black holes. According to the recently updated analysis of wide binaries the permitted mass range is between 30 and 500 solar masses. The present dimensionless entropy of the universe is 1000 googols. This is useful for model building in cyclic cosmology as an alternative to the big bang $[14,15,16,17,18]$. Taking a universe comprised of $10^{11}$ halos each of mass $10^{12} M_{\odot}$, and using a central MACHO mass $100 M_{\odot}$, there are $10^{10}$ MACHOs per halo and $10^{21}$ in the universe. The dimensionless entropy per MACHO, using the PBH formula $[19,20,21]$ is $10^{82}$ and the total is 1000 googols. For galactic-core supermassive black holes (SMBHs), there are $10^{11}$ of them so that for a central mass $10^{7} M_{\odot}$ and individual entropy $10^{92}$ their total entropy is also 1000 googols. 
Because $S \propto M^{2}$, the total mass of MACHOs is much larger than that of SMBHs. For the central values it is $10^{5}$ times larger. The total MACHO mass is $10^{23} M_{\odot}=10^{53} \mathrm{~kg}=$ $10^{80} \mathrm{GeV}$ so I hereby predict several thousand trillion trillion trillion trillion kilograms of black holes!

The existence of such numerous black holes will hopefully be confirmed by observational astronomers. The two most promising known techniques are microlensing and study of wide binaries.

\section{Acknowledgement}

This work was supported by U.S. Department of Energy grant number DE-FG0206ER41418.

\section{REFERENCES}

1. F. Zwicky, Helv. Phys. Acta, 6, 110 (1933).

2. E.W. Kolb and M.S. Turner, The Early Universe, Addison-Wesley (1990).

3. M. Dine, et al. Phys. Lett. 104B, 199 (1981).

4. J.E. Kim, Phys. Rev. Lett. 43, 103 (1979).

5. M.A. Shifman et al., Nucl. Phys. B166, 493 (1980).

6. A.R. Zhitnitsky, Sov. J. Nucl. Phys. 31, 260 (1980).

7. R. Holman, et al. Phys. Lett. B282, 132 (1992).

8. C. Alcock et al. Astrophys. J. 486, 697 (1997).

9. C. Alcock, et al. Astrophys. J. 542, 281 (200)

10. J. Yoo, J. Chaname, and A. Gould, Astrophys.J. 601, 311 (2004).

11. D.P. Quinn, et al. arXiv:0903.1664 [astro-ph]

12. P. Madau and M.J. Rees, Astrophys. J. 551, L27 (2001).

13. J.F. Navarro, C.S. Frenk, and S.D.M. White, Astrophys. J.

14. L. Baum and P.H. Frampton, Phys. Rev. Lett. 98, 071301 (2007).

15. L. Baum and P.H. Frampton, Mod. Phys. Lett. A23, 33 (2008).

16. L. Baum, P.H. Frampton and S. Matsuzaki, JCAP 0804:032 (2008).

17. P.H. Frampton, Mod. Phys. Lett. A22, 2587 (2007)

18. P.H. Frampton, arXiv:0903.4309 [hep-ph] .

19. L. Parker, Phys. Rev. 183, 1057 (1969).

20. J.D. Bekenstein, Phys. Rev. D7, 2333 (1973).

21. S.W. Hawking, Commun. Math. Phys. 43, 1974 (1975). 462, 563 (1996). 